\title{
ESTUDO DO GENE DO HORMÔNIO DE CRESCIMENTO EM UMA POPULAÇÃO DE BÚFALAS DA RAÇA MURRAH (Bubalus bubalis)
}

\section{(A study on the growth hormone gene within a herd of female buffaloes of the Murrah breed (Bubalus bubalis)}

\author{
D'AGOSTINO GARRIDO, D.A. ${ }^{1}$; FERRAZ, A.L.J. ${ }^{2}$ TONHATI, H. ${ }^{3}$;ENNA, J.D. ${ }^{4}$; OTAVIANO, A.R. ${ }^{5}$
}

'Doutora em Zootecnia (Produção Animal) pela Universidade Estadual Paulista Faculdade de Ciências Agrárias e Veterinárias (FCAV/UNESP) campus Jaboticabal. Rua Santo Amaro, 364. CEP:80620-330 Fone

(41) 88342310, debgarrido@hotmail.com. Curitiba, PR. Brasil;

2Doutorando em Zootecnia da FCAV/UNESP, Jaboticabal, SP, Brasil; ${ }^{3}$ Departamento de Zootecnia da Universidade Estadual Paulista Faculdade de Ciências Agrárias e Veterinárias (FCAV/UNESP). Via de Acesso Prof. Paulo Donato Castellane, s/n, CEP 14884-900, Jaboticabal, SP, Brasil; ${ }^{4}$ Departamento de Genética e Melhoramento de Plantas da FCAV/UNESP, Jaboticabal, SP, Brasil. ${ }^{5}$ Doutorando em Zootecnia da FCAV/UNESP, Jaboticabal, SP, Brasil.

\begin{abstract}
RESUMO - O hormônio GH é um dos principais reguladores do crescimento pós-natal e do metabolismo em mamíferos, sendo proposto como gene candidato para identificação de marcadores genéticos relacionados a características de crescimento, carcaça e produção de leite. Dessa forma, o presente estudo objetivou estudar possíveis polimorfismos na parte final da região $5^{\prime}$-flanqueadora, primeiro exon e parte do primeiro intron do gene do hormônio de crescimento de uma população de búfalas da raça Murrah, buscando futuras associações com características de produção de leite e seus constituintes. Para tanto, foram utilizadas amostras de sangue de 115 búfalas. Mediante reação em cadeia da polimerase (PCR), foi amplificado um fragmento com $450 \mathrm{pb}$, o qual foi analisado pela técnica de seqüenciamento e de RFLP (Restriction Fragment Length Polymorphism), utilizando a endonuclease de restrição Ddel. Como resultado, a seqüência de nucleotídeos do gene $\mathrm{GH}$ de búfalos será submetida a GenBank. No entanto, verificou-se que os bubalinos apresentaram monomorfismo, para a região estudada, do gene do hormônio de crescimento. Assim sendo, não foi possível quantificar a ação desse gene sobre a produção de leite e seus constituintes.
\end{abstract}

Palavras-chave: hormônio do crescimento; gene; búfalas.

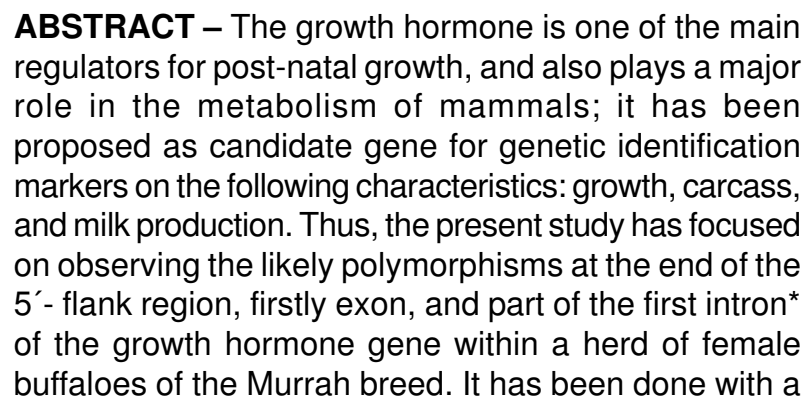

view to spotting future associations, such as characteristics of milk production and its constituents. In order to do that, blood samples of 115 female buffaloes have been used. Through a polimerasis chain reaction, a fragment of $450 \mathrm{pb}$ has been enlarged, and it has been analyzed through the sequencing technique, and through the RFLP, utilizing restriction endonucleasis Ddel. As a result, the sequence of the nucleotides of the growth hormone of the female buffaloes will be submitted to the Genbank. Nevertheless, it has been noted that bubalines have showed monomorphism, in the region which was studied, towards the growth hormone gene. Therefore, it hasn't been possible to quantify the action of this gene on the production of milk and its constituents.

Key-words: growth hormone; gene; Bubalus bubalis.

\section{Introdução}

O hormônio de crescimento $(\mathrm{GH})$ tem sido apontado como um dos principais reguladores do crescimento pós-natal e do metabolismo em mamíferos, afetando assim, a taxa de crescimento, a composição corporal, a saúde e a produção de leite, por meio da modulação da expressão de muitos genes. Portanto, o GH é um gene com grande potencial para estudos de variação molecular em associação com estudos de variação genética de características de interesse econômico (GE et al., 2003).

$\mathrm{O}$ gene $\mathrm{GH}$ bovino (bGH) foi primeiramente seqüenciado por GORDON et al. (1983) e tem sido extensivamente utilizado em experimentos de transferências de genes com animais de laboratório e animais domésticos. De acordo com FRIES et al. (1993), O bGH está localizado no cromossomo 19 e contém aproximadamente 1793 nucleotídeos, apresentando 4 íntrons (regiões não codificadoras) e 5 
Estudo do gene do hormônio de crescimento em uma população de búfalas da raça Murrah (Bubalus bubalis)

exons (regiões codificadoras). A síntese do peptídeo sinal do bGH é direcionada por 26 aminoácidos codificados pelo exon I e parte do exon II, sendo que este peptídeo sinal está relacionado com a secreção do hormônio. A região 5 'flanqueadora é composta de aproximadamente 648 nucleotídeos, contém seqüências curtas de DNA que são reconhecidas por sítios de ligação e de iniciação e que estão associados ao controle da expressão gênica.

O primeiro nível de controle na produção de uma proteína é a regulação da transcrição do gene que a codifica. Nesse sentido, torna-se importante o conhecimento da região promotora, bem como a região 5' não traduzida do gene, visto que, essas regiões possuem vários sítios de ligação de fatores de transcrição, reguladores e inibidores da expressão gênica (WATSON et al., 1997). Polimorfismos nessas regiões do gene $\mathrm{GH}$ bovino foram descritos por $\mathrm{HECHT}$ e GELDERMAN (1996), RODRIGUES et al. (1998); SUZUKI (2001), GE et al. (2003) e FERRAZ et al. (2006).

Considerando que a literatura é escassa no que diz respeito a polimorfismos no gene do hormônio de crescimento em bubalinos é justificável o seu estudo. Assim sendo, o objetivo deste trabalho foi verificar possíveis polimorfismos na região 5'-flanqueadora, primeiro exon e parte do primeiro intron do gene $\mathrm{GH}$ de búfalas da raça Murrah pela técnica de PCR-RFLP e pelo seqüenciamento da referida região.

\section{Material e Métodos}

O estudo foi realizado com 115 búfalas da raça Murrah. Foram coletadas amostras de aproximadamente $5,0 \mathrm{~mL}$ de sangue de cada animal, por punção da veia jugular, utilizando-se de tubos vacutainer contendo $7,5 \mathrm{mg}$ de EDTA. Os tubos contendo as amostras de sangue ficaram sob refrigeração até serem centrifugados a $2700 \times$ g, durante 10 minutos. Em seguida, foi retirado o plasma sangüíneo e a camada de leucócitos foi coletada e armazenada a -20ํㅡ para posterior extração do DNA genômico, conforme metodologia descrita por MIRETTI (1998).

As amostras de DNA foram submetidas à quantificação em gel de agarose $(0,8 \%)$ e o grau de pureza foi determinado em espectrofotômetro (BECKMAN DUÒ640B), segundo SAMBROOK et al. (1989). Após a quantificação, as amostras foram diluídas a $50 \mathrm{ng} / \mathrm{mL}$ e estocadas a $4^{\circ} \mathrm{C}$ para posterior utilização nas reações de amplificação.

Com base na seqüência de nucleotídeos do gene $\mathrm{GH}$ de bovinos depositada no GenBank (gi $1 / 2163091 \frac{1}{2}$ ) foi sintetizado um par de primers, os quais foram desenhados por FERRAZ (2005), para amplificar um fragmento de DNA genômico, que abrange uma parte da região 5'- flanqueadora, o primeiro exon e a parte inicial do primeiro intron do gene $\mathrm{GH}$ de búfalos. As seqüências dos primers estão descritas abaixo:

\section{P3 F: 5' - TCT CAA GCT GAG ACC CTG TGT - 3' \\ P4 R: 5' - GGC CAAATG TCT GGG TGT AGA - 3}

Dessa forma, a seqüência do gene $\mathrm{GH}$ bovino

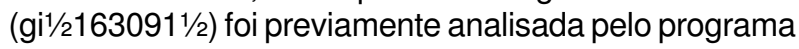
pDRAW 321.0 (www.geocites.com/acaclone), para a reconhecimento da localização dos primers, do tamanho da região a ser amplificada e dos sítios para endonuclease de restrição Ddel (escolhida para análise de RFLP), sendo estes representados pelos símbolos $(+/+),(+/-)$ e (-/-) conforme a presença (+) ou ausência (-) do sitio de restrição (FIGURA 1).

Assim, foi possível observar com a análise virtual, que o tamanho do fragmento da região estudada para o gene GH bovino foi de 453 pb, e que os animais que possuíam o alelo (+) para a enzima Ddel, produziriam os fragmentos de 8 pb, 267 pb e 178 pb.

Além disso, existem duas regiões seqüenciadas do gene $\mathrm{GH}$ de búfalos disponíveis no GenBank, as quais foram submetidas por TIWARI e GRAG (1998) e TIWARI et al. (1998). No entanto, ressalta-se que os trabalhos realizados para a obtenção das referidas seqüências, não foram publicados até o presente momento. Sendo assim, não foi possível encontrar dados sobre a raça e número de animais utilizados para o seqüenciamento nos referidos trabalhos. Além disso, não há um acesso completo para o gene GH bubalino, ou seja, as seqüências disponíveis encontram-se sobrepostas, de modo que foram editadas manualmente, de maneira a se tornar possível uma prévia comparação com a seqüência dos búfalos analisada no presente estudo. Após sobreposição dos acessos a seqüência resultou em um fragmento de 2404 pb, o qual foi também previamente analisado pelo programa pDraw 321.0 (FIGURA 2). 
FIGURA 1 - A) ESQUEMA DO GENE DO GH BOVINO (GI $\left.1 \frac{1}{2} 163091 \frac{1}{2} 2\right)$ EVIDENCIANDO AS POSIÇÕES DOS PRIMERS P3 E P4 QUE FLANQUEIAM A PARTE FINAL DA REGIÃO 5'-FLANQUEADORA, O PRIMEIRO EXON E UMA PARTE DO PRIMEIRO ÍNTRON ALÉM DOS SÍTIOS PARA A ENDONUCLEASE DE RESTRIÇÃO DDEI. B) ESQUEMA EVIDENCIANDO A REGIÃO AMPLIFICADA DO GENE GH BOVINO, E O TAMANHO DOS FRAGMENTOS ESPERADOS PARA O ALELO (+) APÓS DIGESTÃO COM A ENZIMA DDEI.

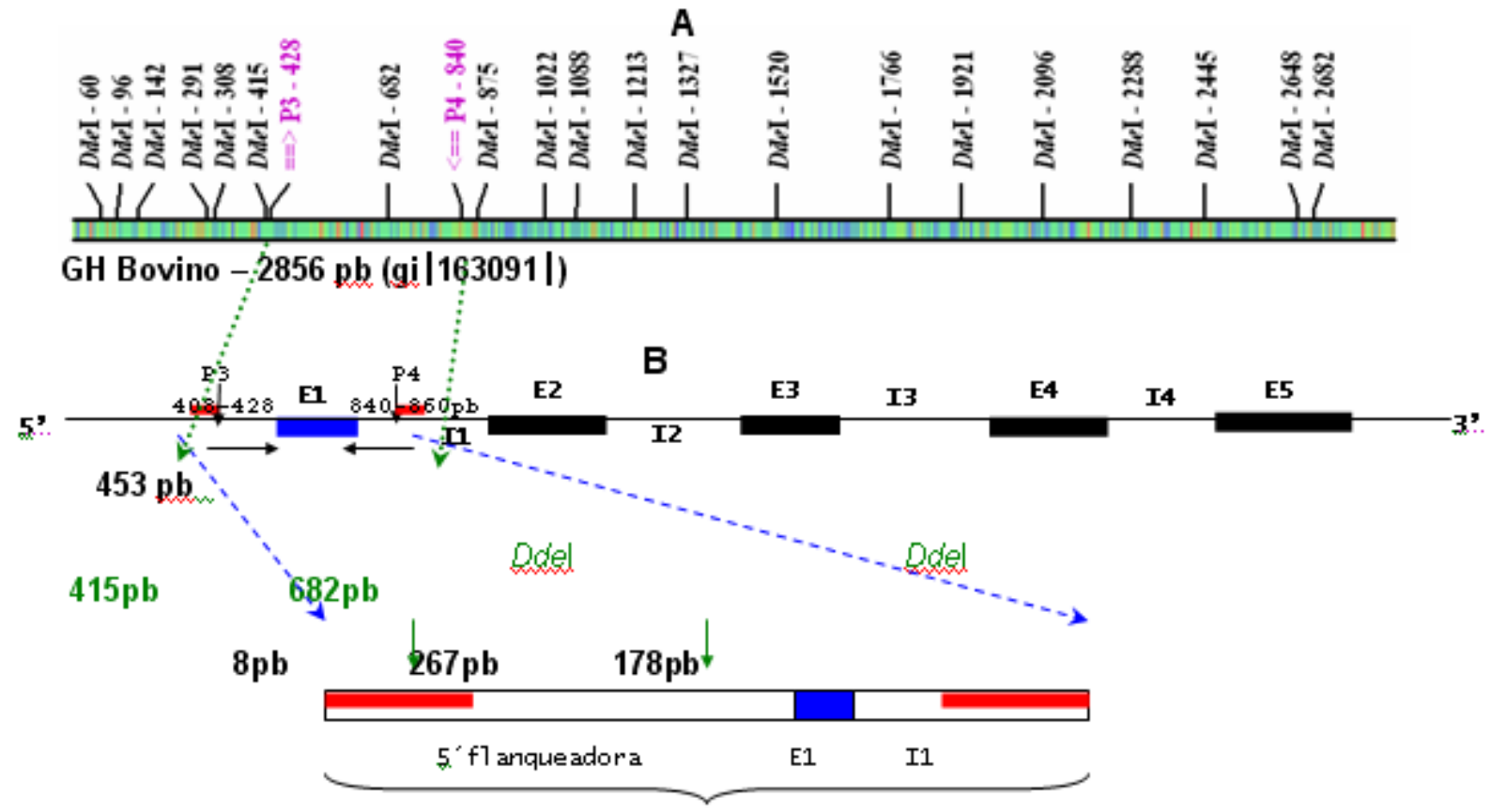

$\mathrm{pb}=$ pares de base

$453 \mathrm{pb}$

FIGURA 2 - A) ESQUEMA DO GENE DO GH BUBALINO (Glú $3046742 u ́$ E Glú 3660482ú ) COM AS POSIÇÕES DOS PRIMERS P3 E P4 QUE FLANQUEIAM A PARTE FINAL DA REGIÃO 5'-FLANQUEADORA, O PRIMEIRO EXON E UMAPARTE DO PRIMEIRO INTRON ALÉM DOS SÍTIOS PARAA ENDONUCLEASE DE RESTRIÇÃO DDEI. B) ESQUEMA EVIDENCIANDO A REGIÃO AMPLIFICADA DO GENE GH BUBALINO, E O TAMANHO DOS FRAGMENTOS ESPERADOS PARA O ALELO (+) APÓS A DIGESTÃO COM A ENZIMA DDEl.

A

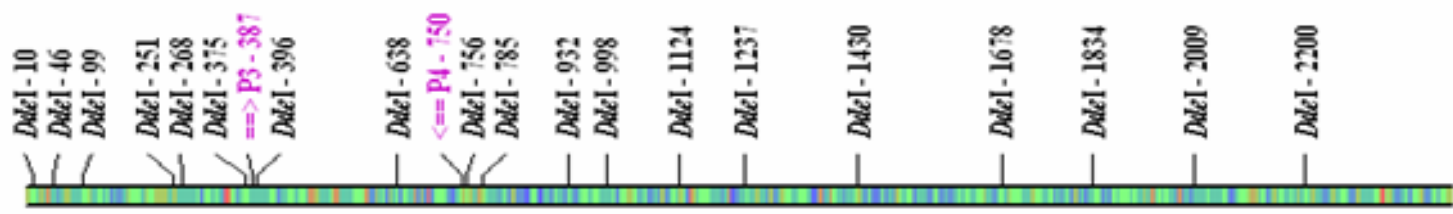

GH bubalino - 2404 ph (gi | 3046742 I e gi | 3660482 l).

B
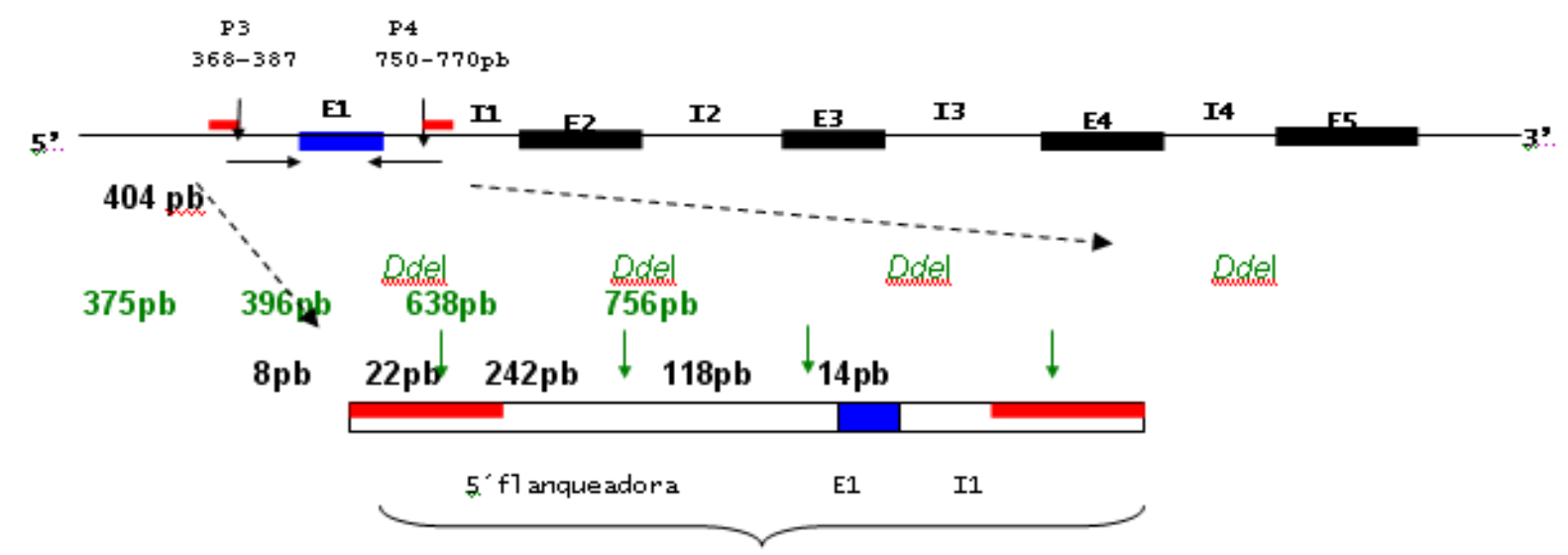

$\mathrm{pb}=$ pares de base $404 \mathrm{pb}$

Archives of Veterinary Science, v.11, n.3, p.36-46, 2006 
Estudo do gene do hormônio de crescimento em uma população de búfalas da raça Murrah (Bubalus bubalis)

Foi possível observar, com essa análise virtual do GH bubalino (giú 3046742ú e giú 3660482ú), que o tamanho do fragmento referente à região estudada foi de 404 pb e que os animais que possuíam o alelo (+) para a enzima Ddel, produziriam os fragmentos de 8 pb, 14 pb, 22 pb, 118 pb e 242 pb.

Após a análise virtual realizada com a seqüência do gene do GH bovino (gi1/21630911/2) e com as seqüências parciais do gene do GH bubalino (giú 3046742ú e giú 3660482ú), as reações de amplificações foram realizadas

O fragmento de DNA dos animais estudados, foi amplificado pela técnica de reação em cadeia de polimerase (PCR), sendo as reações de amplificação realizadas num volume final de $25 \mu \mathrm{L}$, contendo $150 \mathrm{ng}$ de DNA, 0,5 $\mu \mathrm{M}$ de cada primer, $1 \mathrm{X}$ PCR buffer [10 $\mathrm{mM}$ TrisHCl (pH 9,0), 1,5 mM MgCl 2 e $50 \mathrm{mM} \mathrm{KCl}], 100$ $\mu \mathrm{M}$ de dNTPs e 0,5 U Taq DNA polimerase. O programa de amplificação constituiu-se de um ciclo de desnaturação inicial a $94^{\circ} \mathrm{C}$ por 2 minutos, seguido de 35 ciclos de desnaturação a $94^{\circ} \mathrm{C}$ por 1 minuto, anelamento a $59^{\circ} \mathrm{C}$ por 1 minuto e extensão a $72^{\circ} \mathrm{C}$ por 1 minuto. Ao final dos 35 ciclos, os produtos de amplificação foram submetidos a mais um ciclo de extensão a $72^{\circ} \mathrm{C}$ por 15 minutos, para completar a amplificação dos fragmentos e mantidos a $20^{\circ} \mathrm{C}$ para posterior análise.

Os produtos de amplificação foram analisados por meio da técnica de polimorfismo no comprimento do fragmento de restrição (RFLP), sendo $2,5 \mathrm{~mL}$ da reação de amplificação digeridos com a endonuclease Ddel, a $37^{\circ} \mathrm{C}$ por 2,5 horas, e separados por eletroforese (100 V por 1,5 hora de corrida em tampão TBE 1X) em gel de agarose $(3,0 \%)$ corado com brometo de etídio (10 mg/ $\mathrm{mL}$ ). A visualização das bandas foi feita sob luz ultravioleta e o gel foi fotodocumentado (Gel-Doc, Bio-Rad). Para identificação do tamanho dos fragmentos resultantes foi utilizado como referência o padrão de tamanho molecular "1Kb Plus DNA ladder (GIBCO - BRL)".

Para o seqüenciamento da região estudada em búfalos, foram três produtos de amplificação, obtidos de três diferentes animais escolhidos ao acaso. Os produtos amplificados foram clonados separadamente no plasmídeo pGEM-T Easy Vector System I (Promega), conforme a especificação do fabricante.

O produto da ligação $(10 \mu \mathrm{L})$ foi utilizado para transformar células competentes de E. coli da linhagem
DH10B, conforme HANAHAN (1983). Posteriormente, foi realizada a extração dos plasmídeos pelo método de lise alcalina conforme protocolo descrito por SAMBROOK et al. (1989).

O DNA plasmidial extraído de cada clone foi submetido à digestão com a endonuclease de restrição EcoRI $(10 \mathrm{U} / \mathrm{mL})$ a $37^{\circ} \mathrm{C}$ por 2 horas, a fim de que fosse comprovada a presença ou não do inserto de interesse no referido vetor.

Após a confirmação da presença do inserto de interesse, os DNAs plasmidiais dos clones positivos foram submetidos ao seqüenciamento com os primers T7 e SP6 presentes no vetor de clonagem, utilizandose o kit "BigDye ${ }^{\mathrm{TM}}$ Terminator Cycle Sequencing v 3.0" e um seqüenciador automático 3700 DNAAnalyzer (PE Applied Biosystems), conforme as recomendações dos fabricantes.

Para verificar a qualidade das seqüências geradas utilizou-se o programa "Sequencing Analysis 3.4", gerando os eletroferogramas, que foram submetidos à análise pelos programas "Phred/Phrap/Consed" (GORDON et al., 1998).

Posteriormente, para a identificação da presença de polimorfismo, essas seqüências foram alinhadas pelo programa CLUSTAL-X (THOMPSON et al., 1997) sendo comparadas entre si, bem como, com a seqüência do gene $\mathrm{GH}$ bovino (gi $1 / 21630911 \frac{1}{2}$ ), e com as seqüências parciais do $\mathrm{GH}$ bubalino (gi $1 \frac{1}{2} 30467421 / 2$ e gi $1 \frac{1}{2} 3660482 \frac{1}{2}$ ), utilizadas como referência.

\section{Resultados e Discussão}

Os resultados que serão observados a seguir pela análise em gel de agarose (FIGURAS 3 e 4) foram confirmados com o seqüenciamento descrito mais adiante.

A amplificação com os iniciadores P3 e P4 gerou um fragmento com aproximadamente $450 \mathrm{pb}$ para todas as 115 amostras estudadas (FIGURA 3).

O resultado da digestão do fragmento amplificado em búfalos com a enzima Ddel que reconhece a seqüência:

$$
\begin{aligned}
& \text { 5'...C-TNAG...3' } \\
& \text { 3'...GANT-C...5' }
\end{aligned}
$$

gerou os fragmentos de 178 e 242 pb como pode ser visualizado na FIGURA 4. 
FIGURA 3 - FOTO DO GEL EVIDENCIANDO OS PRODUTOS OBTIDOS POR PCR DE AMOSTRAS DE DNA DE ALGUNS DOS ANIMAIS ESTUDADOS, DEMONSTRANDO O FRAGMENTO DE 450 PB, EM GEL DE AGAROSE 1,5\%, PROVENIENTE DAAMPLIFICAÇÃO COM OS INICIADORES P3 E P4. M = MARCADOR MOLECULAR 1KB DNA LADDER (GIBICO - BRL).

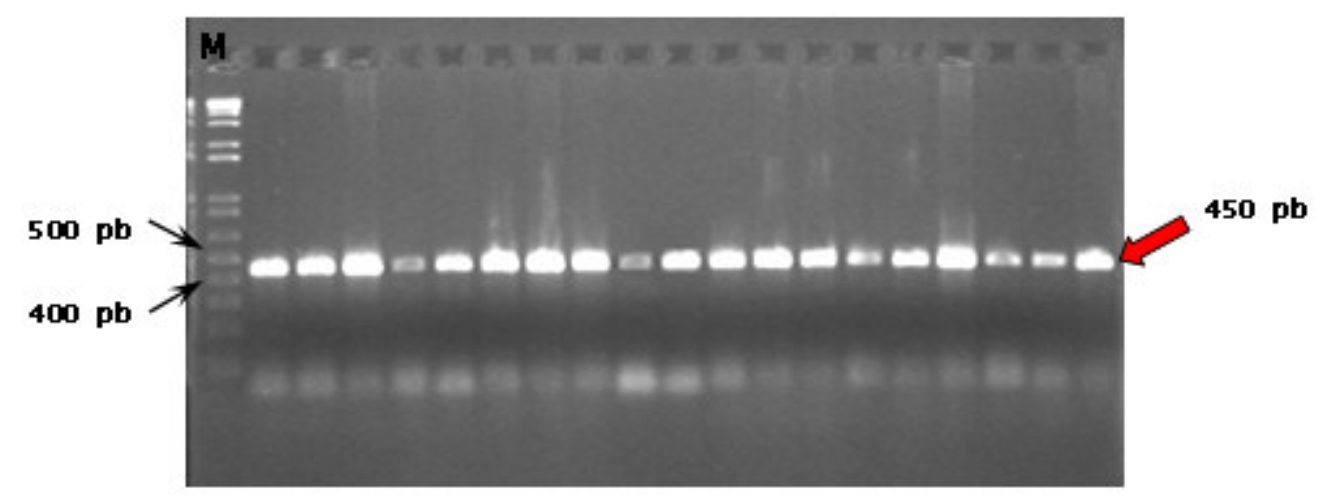

$\mathrm{pb}=$ pares de base

FIGURA 4 - FOTO DO GEL MOSTRANDO O PADRÃO DE MIGRAÇÃO DOS FRAGMENTOS GERADOS APÓS A DIGESTÃO DO FRAGMENTO DE 450 PB COM A ENZIMA DDEI EM GEL DE AGAROSE 3,0\%.M = MARCADOR MOLECULAR 1KB DNA LADDER (GIBICO-BRL).

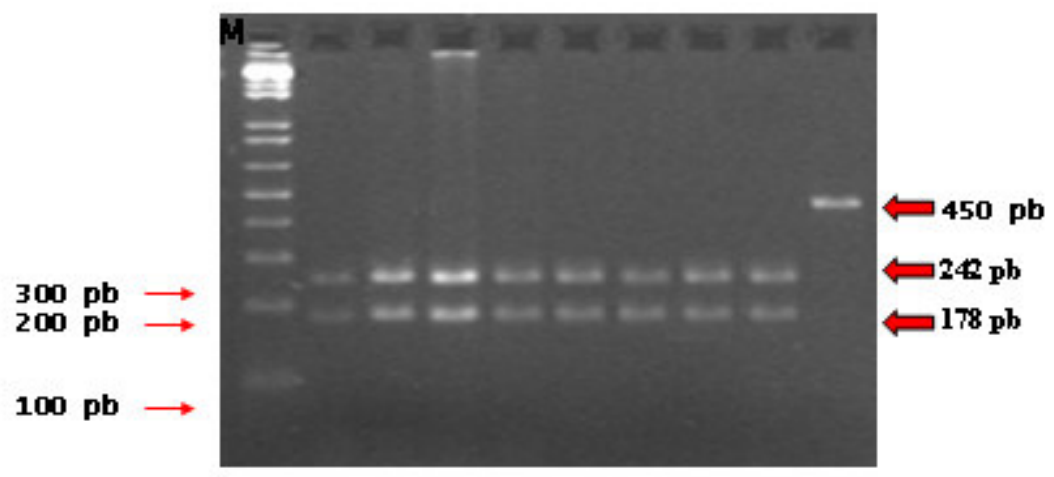

$\mathrm{pb}=$ pares de base

O que se pôde afirmar pela técnica de RFLP é que houve a ocorrência de um único genótipo $(+/+)$ dentro da população estudada, ou seja, a região estudada demonstrou monomorfismo para a enzima Ddel.

Resultados semelhantes de monomorfismo em bubalinos também foram observados por alguns autores (MITRA et al., 1995; MARCHIORI, 2003) que estudando o exon $\mathrm{V}$ do gene $\mathrm{GH}$ de búfalas através da técnica de RFLP, observaram monomorfismo no padrão de migração de fragmentos digeridos com a endonuclease Alul, sugerindo que o monomorfismo encontrado possa ser uma característica da espécie bubalina.

Ao comparar os resultados visualizados na FIGURA 4 com a análise virtual (FIGURA 2), verificou-se que os tamanhos dos produtos amplificados, e dos fragmentos gerados após a digestão com endonuclease Ddel, aparentemente foram semelhantes aos observados por meio da análise virtual em bovinos, considerando que o fragmento de 8 pb não pode ser visualizado em gel de agarose. Por outro lado, ao comparar os resultados visualizados na FIGURA 4 (gel de agarose) com a análise virtual (FIGURA2), verificou-se que os tamanhos dos produtos amplificados, e dos fragmentos gerados após a digestão com endonuclease Ddel, foram diferentes aos observados por meio da análise virtual em bubalinos.

Nesse sentido, foi realizado o seqüenciamento da região estudada em três animais previamente escolhidos com o intuito de confirmar os padrões da mobilidade eletroforética visualizados com o resultado da amplificação e da digestão com a enzima Ddel, bem como verificar possíveis diferenças entre a seqüência dos animais estudados com a seqüência de bovinos

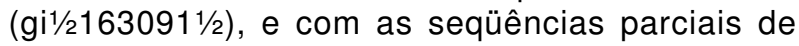
bubalinos (gi $1 / 23046742$ e gi $1 / 236604821 / 2$ ).

Após o seqüenciamento, as três seqüências de nucleotídeos do gene GH dos animais estudados foram alinhadas pelo programa CLUSTAL-X v.1.81 (THOMPSON et al., 1997), comprovando-se que as mesmas não apresentavam diferenças entre si, o que originou uma única seqüência consenso. Esta seqüência consenso foi analisada, primeiramente, pelo programa 
Estudo do gene do hormônio de crescimento em uma população de búfalas da raça Murrah (Bubalus bubalis)

pDRAW 32 1, cujo resultado pode ser visualizado na FIGURA 5.

Por meio dessa análise verificou-se que o fragmento seqüenciado, o qual corresponde à região estudada, apresentou 450 pb e os animais que possuem o alelo (+) para a enzima $D d e l$ produziram os fragmentos de $8 \mathrm{pb}, 22 \mathrm{pb} 178 \mathrm{pb}$ e 242 pb, confirmando-se assim os resultados observados pela análise em gel de agarose $3 \%$, uma vez que os fragmentos de 8 pb e 22 pb não foram visualizados, devido ao baixo poder de resolução do gel. Logo, os dois fragmentos maiores (178 e $242 \mathrm{pb}$ ) foram confirmados pelo seqüenciamento.

FIGURA 5 - ESQUEMA EVIDENCIANDO A ANÁLISE DA REGIÃO CONSENSO SEQÜENCIADA, REFERENTE AOS ANIMAIS ESTUDADOS (BÚFALOS), COM AS POSIÇÕES DOS PRIMERS E O TAMANHO DOS FRAGMENTOS ESPERADOS PARA O ALELO (+), APÓS DIGESTÃO COM A ENZIMA DDEI.

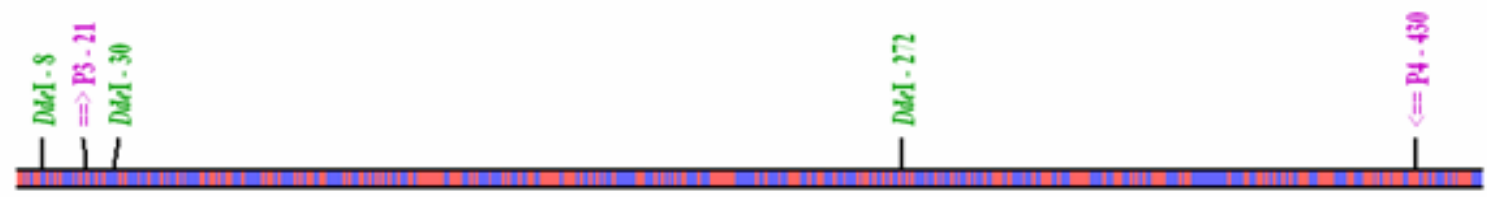

bubalino - sequiência consenso 450 pb

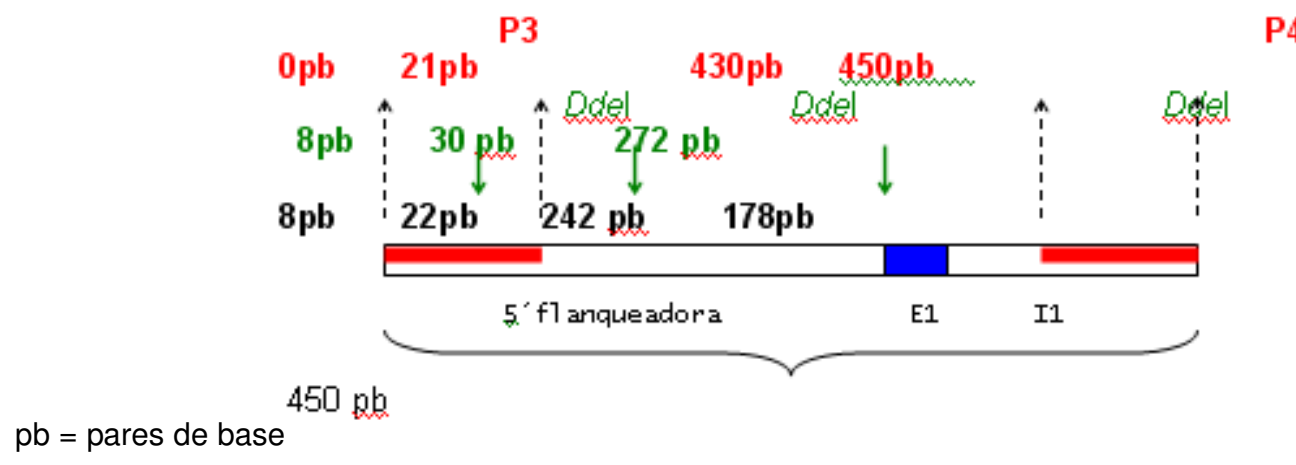

P4

SUZUKI (2001) e FERRAZ (2003) observaram a existência de dois alelos para o fragmento $\mathrm{GH}$ bovino correspondentes à região pesquisada nesse trabalho e nenhum polimorfismo encontrado estava posicionado na região codificadora do gene do $\mathrm{GH}$, constituíndo-se em mutações silenciosas.

Em seguida, as referidas seqüências foram alinhadas e comparadas com a seqüência de nucleotídeos da região estudada do gene GH bovino (gi1/21630911/2) pelo programa CLUSTAL-X v.1.81 (THOMPSON et al., 1997). Mediante essa análise de comparação foi possível observar que a região estudada do $\mathrm{GH}$ em búfalos apresentou $95 \%$ de homologia com a seqüência de bovino e que o fragmento do gene do $\mathrm{GH}$ dos animais seqüenciados apresentou treze variações em relação à seqüência de bovinos (FIGURA 6).

Assim sendo, quando comparada à seqüência de bovinos (gi 1/21630911/2), o fragmento seqüenciado não apresentou duas adeninas e uma guanina (AAG), nas posições 614 pb, 615 pb e 616 pb. Essa diferença é a razão pela qual o tamanho do fragmento seqüenciado (450 pb) é diferente do observado em bovinos (453 pb). A deleção dessas três bases, localizada nas proximidades da seqüência TATAA foi identificada por RODRIGUES et al. (1998) e FERRAZ (2006) que estudaram a região promotora, o exon I e o intron I no gene bGH.

Avariação $A / T$ que ocorre, na seqüência obtida, na posição 440 pb, está dentro de um sítio de restrição para endonuclease Ddel. Esta ocasiona a perda do sítio para a referida enzima nos bovinos. Assim, constatou-se que a seqüência dos animais em estudo apresenta 3 sítios de restrição Ddel (posições 415, 440 e 682 pb) e a seqüência de bovinos apresenta 2 sítios (posições 415 e 682 pb).

Apesar do número e do tamanho dos fragmentos digeridos serem diferentes entre os animais estudados e os bovinos, uma avaliação baseada apenas no resultado de digestão, visualizada em gel de agarose, não poderia ser comprovada, visto que restaria dúvidas sobre a presença ou ausência dos fragmentos de 8 pb e 22 pb provenientes da digestão nos animais em estudo. Portanto, somente o seqüenciamento pôde confirmar o resultado obtido pela análise de RFLP.

Além disso, com o intuito de se verificar possíveis diferenças entre os animais da espécie Bubalus bubalis, a seqüência consenso obtida nesse estudo foi comparada à seqüência de búfalos (gi $1 \frac{1}{2} 30467421 \frac{1}{2}$ e gi $1 \frac{1}{2} 36604821 / 2$ ) por meio do programa CLUSTAL-X v.1.81 (THOMPSON et al., 1997), sendo apontadas 49 diferenças (FIGURA 7).

Verificou-se presença de 46 bases na região do intron I, o que pode corresponder a um "gap" na seqüência de búfalos depositada no banco de dados. Essas variações ocasionam uma diferença em relação ao tamanho do fragmento amplificado, ou seja, o fragmento seqüenciado no presente trabalho possui $450 \mathrm{pb}$ (FIGURAS 3 e 5) e o fragmento depositado no banco de dados possui $404 \mathrm{pb}$ (FIGURA 2). 
FIGURA 6 - ALINHAMENTO DAS SEQÜÊNCIAS DE NUCLEOTÍDEOS REFERENTE À REGIÃO ESTUDADA DO GENE GH DOS ANIMAIS SEQÜENCIADOS E DE BOVINOS (GI 1/21630911/2), PELO CLUSTAL-X V.1.81 EVIDENCIANDO AS DIFERENÇAS ENTRE AS MESMAS.

GH1 fasta.screen.Contig1 - Ddel Ddel $\quad \downarrow$

QH2 fasta.screen.Contig1 -

GGCCGCGGGAATTCGATTTCTCAAGCTGAGACCCTGTGTGCACAGCCCT 49

GH4, fasta.screen.Contig1 -

GGCCGCGGGAATTCGATTTCTCAAGCTGAGACCCTGTGTGCACAGCCCT 49

GHFASTA.BOVINE AAACCTATGGGGT--GGGCTCTCAAGCT GAGACCCT GTGTGCACAGCCCT 438

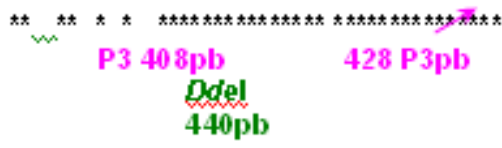

GH1 fasta.screen Contig1 CAGGCTGGTGGCAGTGGAGAGGGGATGATGACAAGCCTGGGGGACATGAC 99 GH2,fasta. screen.Contig1 CAGGCTGGTGGCAGTGGAGAGGGGATGATGACAAGCCTGGGGGACATGAC 99 GH4 fasta. screen. Contig1 CAGGCTGGTGGCAGTGGAGAGGGGATGATGACAAGCCTGGGGGACATGAC 99 GHFASTA.BOVINE

CTGGCTGGTGGCAGTGGAGACGGGATGATGACAAGCCTGGGGGACATGAC 488

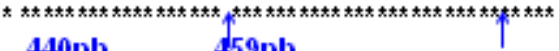

$440 \mathrm{pb} \quad 459 \mathrm{pb}$

QH1 fasta.screen.Contig1

CCCAGAGAAGGAACGGGAACAGGATGAGTGAGAGGAGGTTCTAAATTATC 149 QH2,fasta screen.Contig1 CCCAGAGAAGGAACGGGAACAGGATGAGTGAGAGGAGGTTCTAAATTATC 149 GH4 fasta. screen.Contig1 CCCAGAGAAGGAACG GGAACAGGATGAGTGAGAGGAGGTTCTAAATTATC 149 GHFASTA.BOVINE

CCCAGAGAAGGAACGGGAACAGGATGAGTGAGAGGAGGTTCTAAATTATC 538

\begin{tabular}{|c|c|}
\hline H1 fasta screen Contig1 & 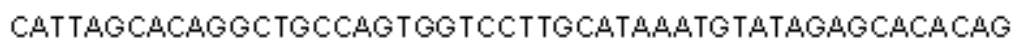 \\
\hline fasta.screen.Contig1 & САTTAGCACAGGCTGCCAGTGGTCСTTGCATAAATGTATAGAGCACACAG \\
\hline H4 fasta. screen.Contig1 & САTTAGCACAGGCTGCCAGTGGTCСTTGCATAAATGTATAGAGCACACAG \\
\hline FASTA.BOVINE & 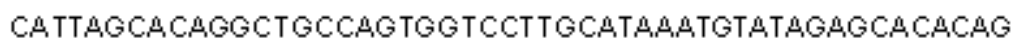 \\
\hline
\end{tabular}
588

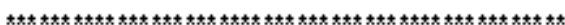

GH1 fasta. screen.Contig1 GTGGGGGGAAAGGGAGAGAGAGAA--GCCAGGGTATAAAAAGGGCCCAG 246

GH2,fasta. screen Contig1 GTGGGGGGAAAGGGAGAGAGAGAA--GCCAGGGTATAAAAAGGGCCCAG 246

GH4 fasta.screen.Contig1 GTGGGGGGAAAGGGAGAGAGAGAA-GCCAGGGTATAAAAAGGGCCCAG 246 
Estudo do gene do hormônio de crescimento em uma população de búfalas da raça Murrah (Bubalus bubalis)

GHFASTA.BOVINE

GTGGGGGGAAAGGGAGAGAGAGAAGAAGCCAGGGTATAAAAATGGCCCAG 638

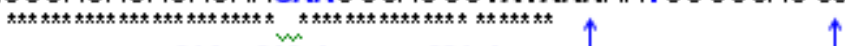
$614-616 \mathrm{pb} \quad 631 \mathrm{pb}$

Ddel

QH1 fasta.screen.Contig1

CAGGGACCAATT CCAGGATCCCAGGACCCACT TCACCAGACGACTCAGGG 296

GH2,tasta.screen Contig1

CAGGGACCAATTCCAGGATCCCAGGACCCACTTCACCAGACGACTCAGGG 296

GH4 fasta.screen Contig1

CAGGGACCAATTCCAGGATCCCAGGACCCACTTCACCAGACGACTCAGGG 296 GHFASTA.BOVINE

CAGGGACCAATT CCAGGATCCCAGGACCCAGTTCACCAGACGACTCAGGG $688 \uparrow$

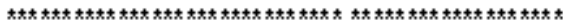

$669 \mathrm{pb} \quad$ odel

EXON 1

$682 p b$

QH1 fasta. screen.Contig1

M.M A A

TCCTGTGGACAGCTCACCAGCTATGATGGCTGCAGGTAAGCTCGCTAAAA 346 GH2.fasta.screen.Contig1

TCCTGTGGACAGCTCACCAGCTATGATGGCTGCAGGTAAGCTCGCTAAAA 346

GH4 fasta. screen.Contig1

TCCTGTGGACAGCTCACCAGCTATGATGGCTGCAGGTAAGCTCGCTAAAA 346

GHFASTA.BOVINE

TCCTGTGGACAGCTCACCAGCTATGATGGCTGCAGGTAAGCTCGCTAAAA 738

QH1 fasta screen Contig1

TCCCCTCCATTCGTGTGTCCTAAAGGGGTGATGCGGGGGGCCCTGCCGAT 396 GH2.fasta.screen.Contig1

TCCCCTCCATTCGTGTGTCCTAAAGGGGTGATGCGGGGGGCCCTGCCGAT 396

GH4 fasta.screen.Contig1

TCCCCTCCATTCGTGTGTCCTAAAGGGGTGATGCGGGGGGCCCTGCCGAT 396

GHFASTA.BOVINE

TCCCCTCCATTCGCGTGTCCTAAAGGGGTAATGCGGGGGGCCCTGCCGAT 788

$\mathrm{C}$

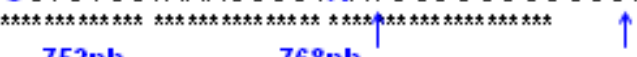

$752 \mathrm{pb}$

$768 \mathrm{pb}$

GH1 fasta.screen.Contig1 GGATGTGTCCACAGCTTTGGGTTTTAGGGCTTCCGAATGTGAACATAGGT 446

GH2.fasta. screen.Contig1

446

GH4 fasta.screen.Contig1

446

GHFASTA.BOVINE

GATGTOTCCACAGCTTTGGGTTTTAGGGCTTCCGAATGTGAACATAGGT

GGATGTGTCCACAGCTTTGGGTTTTAGGGCTTCCGAATGTGAACATAGGT 838 GGATGTGTTCAGAGCTTTGGGCTTTAGGGCTTCCGAATGTGAACATAGGT

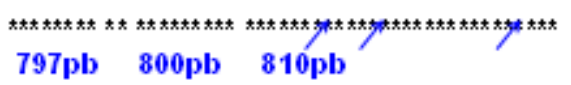

797 pb 800 pb 810 pb

GH1 fasta.screen.Contig1 QH2 fasta. Screen.Contig1 GH4 fasta.screen.Contig1 GHFASTA.BOVINE

ATCTACACCCAGACATTTGGCCAATCACTAGTGAATTC ATCTACACCCAGACATTTGGCCAATCACTAGTGAATTC 484 ATCTACACCCAGACATTTGGCCAATCGCGAGCGAATCC 484 868

ATCTACACCCAGACATTTGGCCAAGTTTGAAATGTTCTCAGTCCCTGGAG

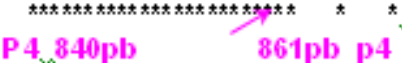


FIGURA 7 - ALINHAMENTO DAS SEQÜÊNCIAS DE NUCLEOTÍDEOS REFERENTE À REGIÃO ESTUDADA DO GH DA SEQÜENNCIA CONSENSO DOS ANIMAIS SEQÜENCIADOS NO PRESENTE TRABALHO E A

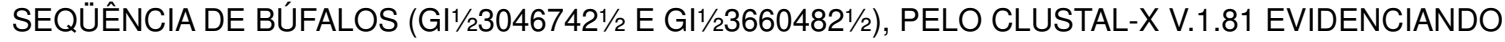
AS DIFERENÇAS ENTRE AS MESMAS.

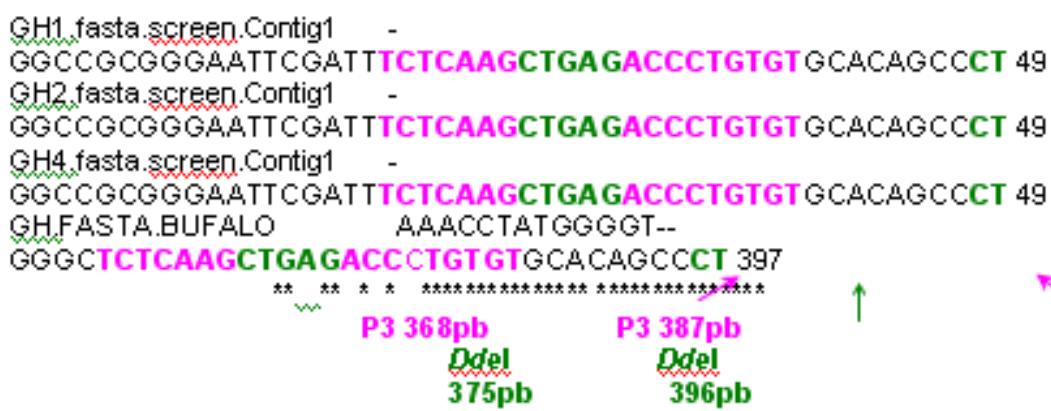

GH1.fasta.screen.Contig1 CAGGCTGGTGGCAGTGGAGAGGGGATGATGACAAGCCTGGGGGACATGAC 99 GH2.fasta. screen.Contig1 CAGGCTGGTGGCAGTGGAGAGGGGATGATGACAAGCCTGGGGGACATGAC 99 GH4 , fasta.screen.Contig1 CAGGCTGGTGGCAGTGGAGAGGGGATGATGACAAGCCTGGGGGACATGAC 99 GHFASTA.BUFALO CAGGCTGGTGGCAGTGGAGAGGGGATGATGACGAGCCTGGGGGACATGAC 447

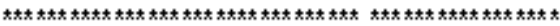
$430 \mathrm{pb}$

GH1 fasta.screen.Contig1 CCCAGAGAAGGAACGGGAACAGGATGAGTGAGAGGAGGTTCTAAATTATC 149 QH2.fasta.screen.Contig1 CCCAGAGAAGGAACGGGAACAGGATGAGTGAGAGGAGGTTCTAAATTATC 149 $\mathrm{GH} 4$, fasta. screen.Contig1 CCCAGAGAAGGAACGGGAACAGGATGAGTGAGAGGAGGTTCTAAATTATC 149 GHFASTA.BUFALO

GH1 fasta screen Contig1 CATTAGCACAGGCTGCCAGTGGTCCTTGCATAAATGTATAGAGCACACAG 199

GH2,fasta. screen.Contig1 CATTAGCACAGGCTGCCAGTGGTCCTTGCATAAATGTATAGAGCACACAG 199

GH4, fasta. screen Contig1 CATTAGCACAGGCTGCCAGTGGTCCTTGCATAAATGTATAGAGCACACAG 199

GHFASTA.BUFALO

CATTAGCACAGGCTGCCAGTGGTCCTTGCATAAATGTATAGAGCACACAG 547

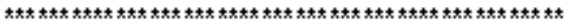

GH1 fasta.screen.Contig1 GTGGGGGGAAAGGGAGAGAGAGAAGCCAGGGTATAAAAAGGGCCCAG 246

GH2.fasta.screen.Contig1 GTGGGGGGAAAGGGAGAGAGAGAAGCCAGGGTATAAAAAGGGCCCAG

246

QH4 fasta.screen.Contig1 GTGGGGGGAAAGGGAGAGAGAGAAGCCAGGGTATAAAAAGGGCCCAG 246 GHFASTA.BUFALO GTGGGGGGAAAGGGAGAGAGAGAAGCCAGGGTATAAAAAGGGCCCAG 594 
Estudo do gene do hormônio de crescimento em uma população de búfalas da raça Murrah (Bubalus bubalis)

GH1 fasta. screen.Contig1

Ddd

CAGGGACCAATTCCAGGATCCCAGGACCCACTTCACCAGACGACTCAGGG 296

QH2 fasta screen.Contig1

CAGGGACCAATTCCAGGATCCCAGGACCCACTTCACCAGACGACTCAGGG 296

GH4 fasta screen Contig1

CAGGGACCAATTCCAGGAT CCCAGGACCCACTTCACCAGACGACTCAGGG 296 GHFASTA.BUFALO

CAGGGACCAATTCCAGGATCCCAGGACCCACT TCACCAGACGACTCAGGG 644

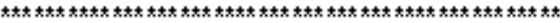

Ddd

EXON 1

$638 \mathrm{pb}$

QH1. fasta.s 8 een.Contig1

$M$ M A A

TCCTGTGGACAGCTCACCAGCTAT GATGGCTGCAGGTAAGCTCGCTAAAA 346

GH2.fasta screen.Contig1

TCCTGTGGACAGCTCACCAGCTAT GATGGCTGCAGGTAAGCTCGCTAAAA 346

GH4. fasta. screen.Contig1

TCCTGTGGACAGCTCACCAGCTAT GATGGCTGCAGGTAAGCTCGCTAAAA 346

GHFASTA.BUFALO

TCCTGTGGACAGCTCACCAGCTATGATGGCTGCA GGTAAGCTCGCTAAAA, 694

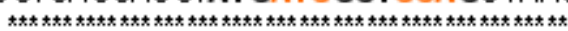

GH1 fasta.screen.Contig1

TCCCCTCCATTCGTGTGTCCTAAAGGGGTGATGCGGGGGGCCCTGCCGAT 396

GH2,fasta.screen Contig1

TCCCCTCCATTCGTGTGTCCTAAAGGGGTGATGCGGGGGGCCCTGCCGAT 396

GH4 fasta screen.Contig1

TCCCCTCCATTCGTGTGTCCTAAAGGGGTGATGCGGGGGGCCCTGCCGAT 396

GHFASTA BUFALO

TCCCCTCCATTCGTGTGTCCGAAAGGGGTGATGCGGGGGGCCCTGCCGAT 744 *t***********************************************

$715 \mathrm{pb}$

GH1.fasta.screen.Contig1 GGATGTGTCCACAGCTTTGGGTTTTAGGGCTTCCGAATGTGAACATAGGT 446

QH2,fasta.screen.Contig1

446

GH4. fasta screen .Contig1 446

GHFASTA BUFALO

GGATGTGTCCACAGCTTTGGGTTTTAGGGCTTCCGAATGTGAACATAGGT

GGATGTGTCCACAGCTTTGGGTTTTAGGGCTTCCGAATGTGAACATAGGT

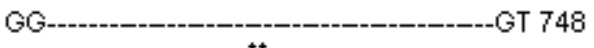

GH1 fasta.screen.Contig1

QH2,fasta.screen Contig1

QH4. fasta screen.Contig1

ATCTACACCCAGACATTTGGCCAATCACTAGTGAATTC-

ATCTACACCCAGACATTTG GCCAAT CGCGAGCGAATCC-------- 484 GHFASTA.BUFALO

ATCTACACTCAGACATTTGGCCAAGTTTGAAATGTTCTCAGTCCCTGGAG 798

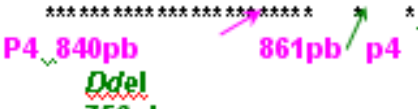


A seqüência dos animais estudados apresenta uma Citosina na posição 756 pb, enquanto que a seqüência dos búfalos depositada no GenBank apresenta uma Timina na mesma posição. Essa variação $\mathrm{C} / \mathrm{T}$ ocasionaria a perda do sitio de restrição da endonuclease Ddel na seqüência dos animais estudados. No entanto, pelo fato da referida variação se encontrar dentro do iniciador P4, não deve ser levada em consideração. Sendo assim, provavelmente uma avaliação previa da análise de digestão do fragmento estudado, em gel de agarose (FIGURA 4), baseada numa análise virtual (FIGURA 2) da seqüência de nucleotídeos dos bubalinos (gi $1 / 230467421 / 2$ e gi $1 / 23660482 \frac{1}{2}$ ) ocasionaria duvidas sobre o perfil da mobilidade eletroforética devido as diferenças dos tamanhos dos fragmentos digeridos, uma vez que a seqüência dos animais estudados e seqüenciados apresenta 3 sítios de restrição Ddel (posições 375, 396 e 638 pb) os quais produzem os fragmentos de 8, 22, 178 e 242 pb, e a seqüência de búfalos depositada no GenBank apresenta virtualmente 4 sítios Ddel (posições 375, 396, 638 e 756 pb) gerando os fragmentos de 8, 14, 22, 118 e 242 pb.

A seqüência de nucleotídeos do gene $\mathrm{GH}$ dos búfalos que abrange uma parte da região 5'- flanqueadora, o primeiro exon e a parte inicial do primeiro intron do gene GH de búfalos, será publicada no GenBank uma vez que a mesma apresentou diferenças quando comparada com a seqüência de búfalos já depositada por TIWARI et al. (1998) e TIWARI e GRAG (1998).

\section{Conclusões}

Os resultados obtidos pela técnica de RFLP evidenciaram monomorfismo no sítio da endonuclease Ddel, sendo portanto todos os animais estudados pertencentes ao genótipo (+/+).

\section{Referências}

FERRAZ, A.L.J. Interações entre polimorfismos no gene do hormônio de crescimento, níveis palsmáticos de IGF-1 e características de carcaça em bovinos de corte. Dissertação (Mestrado na Produção Animal), 2003, 50f. Faculdade de Ciências Agrárias e Veterinárias - UNESP. Jaboticabal. SP. 2003.

FERRAZ, A.L.J.; BORTOLOSSI, J.C.; CURI, R.A.; FERRO, M.I.T.; FERRO, J.A.; FURLAN, N.L.R. Identification and charazterization of polymorphisms within the $5^{\prime}$ flanking region, first exon and part of first intron of bovine $\mathrm{GH}$ gene. Journal of Animal Breeding Genetics., v.123, p.208-212, 2006.

FRIES, R.; EGGEN, A.; WOMACK, J.E. The genome map. Mammalian Genome, v.4, p.405, 1993.

GE, W. DAVIS, M.E.; HINES, H.C.; IRVIN, K.M.; SIMMEN, R.C.M. Association of single nucleotide polymorphisms in the growth hormone receptor genes with serum insulinlike growth factor I concentration and growth traits in Angus cattle. Journal of Animal Science, v.8, p.641-648, 2003.
GORDON, D.; ABAJIAN, C.; GREEN, P. Consed: A graphical tool for sequence finishing. Genome Research, v.8, p.195-202, 1998.

GORDON, D.F.; QUICK, D.P.; ERWIN C.R.; DONELSON, J.E.; MAURER R.A. Nucleotide sequence of the bovine growth hormone chomossomal gene. Molecular and Cellular Endocrinology, v 33, p 81-85, 1983.

HANAHAN, D. Studies on transformation of Escherichia coli with plasmids. Journal Molecular Biologic v.166 (4), p. 557-580, 1983.

HECHT, C.; GELDERMAN, H. Variants within the $5^{\prime}$ flanking region and the intron I of the bovine growth hormone gene. Animal Genetics., v.27, p.329-332, 1996.

MARCHIORI, K.J. Identificação de polimorfismos no 5 o exon do gene do hormônio de crescimento (GH) em búfalas, por PCR-RFLP, 2003. 60f. Trabalho de Graduação em Zootecnia. Faculdade de Ciências Agrárias e Veterinárias - UNESP. Jaboticabal. SP. 2003.

MIRETTI, M.M. Variabilidade genética no lócus BoLADRB3.2 de bovinos nativos e exóticos. Dissertação (Mestrado em Genética) - Faculdade de MeLclustawdicina, Universidade de São Paulo, Ribeirão Preto, 1998.

MITRA, A. Polymorphism at growth-hormone and prolactin loci in Indian cattle and buffalo. Journal of Animal Breeding Genetics, v. 112, p.71-74, 1995.

RODRIGUES, C.V.; GUIMARAES, S.E.; DIAS NETO, E.; PINHEIRO, L.E. Identification of novel polymorphism in the promoter region of the bovine growth hormone gene. Animal Genetics, v.29, p.63-71, 1998.

SAMBROOK, J.; FRITSCH, E.F.; MANIATIS, T. Molecular Cloning: a laboratory manual. $2^{a}$ ed. New York: Cold Spring Harbor Laboratory Press, 1989. 626p.

SUZUKI, J.J. Identificação de polimorfismo associados a características produtivas na região do promotor do gene do hormônio de crescimento em bovinos e utilização como possível marcador molecular. 2001. 61f. Dissertação (Mestrado em Produção Animal) Faculdade de Ciências Agrárias e Veterinária - Universidade Estadual Paulista, Jaboticabal, 2001.

TIWARI, G.; GRAG, L.C. Bubalus bubalis. Encoding growth hormone, exon 1-5. GenBank Assecion No (gi $\left.1 \frac{1}{2} 3660482 \frac{1}{2}\right)$, 1998.

TIWARI, G.; DAS, P.; GRAG, L.C. Bubalus bubalis. Encoding growth hormone, promoter region. GenBank Assecion No (1/236604821/2, 1998.

THOMPSON, J.D.; GIBSON, T.J.; PLEWNIAK, F.; JEANMOUNGIN, F.; HIGGINS, D.G. The CLUSTAL-X windows interface: flexible strategies for multiple sequence alignment aided by quality analysis tools. Nucleic Acids Research, p.4876-4882, 1997.

WATSON, J.D.; GILMAR, M.; J;WIKOWSKI, J. ; Zoller, M., O DNA Recombinante. $2^{2}$. ed. Ouro Preto: Editora EEOP, 1997. 624p.

Recebido para publicação: Aprovado: $26 / 10 / 2006$ 